\title{
Evaluation, identification and impact assessment of abnormal internal standard response variability in regulated LC-MS bioanalysis
}

\author{
Yunlin Fu*,1, Deborah Barkley ${ }^{1}$, Wenkui Li ${ }^{1}$, Franck Picard ${ }^{2} \&$ Jimmy Flarakos ${ }^{1}$ \\ ${ }^{1}$ Pharmacokinetic Sciences, Novartis Institutes for BioMedical Research, East Hanover, NJ 07936, USA \\ ${ }^{2}$ Pharmacokinetic Sciences, Novartis Institutes for Biomedical Research, CH-4056 Basel, Switzerland \\ *Author for correspondence: Tel.: +1 862778 1726; Fax: +1 973781 7579; yunlin.fu@novartis.com
}

Internal standard (IS) plays an important role in LC-MS bioanalysis by compensating for the variability of the analyte of interest in bioanalytical workflow. Due to the complexity of biological sample compositions and bioanalytical processes, a certain level of IS response variability across a run or a study is anticipated. However, an extensive variability may raise doubts to the accuracy of the measured results and also suggest nonoptimal analytical method. In this current paper, recent publications and guidelines regarding IS response in LC-MS bioanalysis were thoroughly reviewed with focus on the evaluation, identification and impact assessment of 'abnormal' Is response variability. A systematic decision tree was proposed to facilitate investigation into abnormal IS response variability after each run.

First draft submitted: 3 March 2020; Accepted for publication: 9 April 2020; Published online: 30 April 2020

Keywords: bioanalysis $\bullet$ impact assessment $\bullet$ internal standard $\bullet$ investigation $\bullet$ LC - MS $/$ MS $\bullet$ root cause $\bullet$ variability

Internal standard (IS) has been widely employed in quantitative liquid chromatography-tandem mass spectrometry (LC-MS/MS) bioanalysis in support of drug discovery and development and post approval therapeutic drug monitoring [1,2]. In practice, a fixed amount of IS is added to zero samples (blank plus IS only), calibration standards (Cs), quality controls (QCs) and study samples in each bioanalytical run. Genarally, the IS goes through exactly the same sample extraction, injection, chromatographic separation, and mass spectrometric detection [3,4] to compensate for the variation that occurs to the analyte of interest during these processes. A proper IS should have its physicochemical properties very close to the analyte of interest, thus behaves similarly or identically as the analyte for optimal response normalization. Ideally, a stable-isotope labeled internal standard (SIL IS) is preferred whenever possible, as it has exactly the same structure as the analyte and co-elutes with it. Under a situation where SIL IS is not available, a structural analog or other molecule may be used [5] as long as it tracks the analyte of interest in the bioanalytical process [6]. Equally important, an appropriate employment of IS is essential for the robustness of the intended bioanalytical assay [1].

In reality, due to the complexity of LC-MS bioanalytical processes, a certain level of IS response variability across a bioanalytical run or a study is more or less anticipated. In some cases, excess variability can be encountered due to subject sample-specific reason(s), human error(s) and/or instrument malfunction [7-10]. In the past years, much discussion has taken place in the bioanalytical community on IS selection, IS employment in bioanalytical process, IS response monitoring, root cause of abnormal IS response and associated investigation, assessment of possible impact due to abnormal IS response, and remediation of impacted results due to abnormal IS response [9-17]. The requirements and recommendations on IS performance in bioanalysis have been well considered by various regulatory bodies. These were reflected in the respective bioanalytical method validation guidelines [16-18]. In Sep-2019, the US FDA issued a guidance for industry titled 'Evaluation of Internal Standard Responses during Chromatographic Bioanalysis: Questions and Answers'. This document contains some detailed recommendations 
on the variability of IS response in chromatographic analytical data when new applications or biological licensing are submitted [15].

In the current review on evaluation, identification and impact assessment of 'abnormal' IS response variability in LC-MS bioanalysis, selection of IS, best practice of IS employment, IS response monitoring, common root causes of abnormal IS response variability and associated investigation, and assessment of possible impact due to abnormal IS response variability were comprehensively re-visited. A systematic decision tree was proposed to investigate abnormal IS response variability after each sample analysis run. Remediation of troubling bioanalytical results due to abnormal IS response variability is beyond the scope of the current review.

\section{IS response variability in method development \& pre-study validation}

The tracking ability of an IS is a key factor that affects the overall performance of an LC-MS/MS assay. A proper IS is expected to compensate for the variability of the analyte of interest across all three stages of LC-MS bioanalytical processes, including sample extraction, chromatographic separation and mass spectrometric detection. For a robust method, if the same amount of the IS is introduced to each sample (Cs, QCs and study samples) and the same amount/volume of sample extract is injected to the LC-MS system, the IS response is expected to be relatively constant between the knowns (Cs and QCs) and unknowns (study samples) cross the entire run.

\section{Selection of an appropriate IS}

An appropriate IS should have the following properties: similar physicochemical properties as the analyte of interest [5], structural uniqueness from endogenous compounds, co-elution with the analyte of interest and MS resolution from the analyte of interest and possible interfering compounds [14]. The closer structurally an IS is to the analyte of interest, the better it tracks the analyte in LC-MS bioanalysis. SIL ISs, such as ${ }^{2} \mathrm{H}-,{ }^{13} \mathrm{C}-$, and ${ }^{15} \mathrm{~N}$-labeled analytes, with nearly identical physicochemical properties but a different mass than the analyte, are the best choices and have been extensively employed in the regulated LC-MS bioanalysis [19-22]. Other advantages of SIL IS have been reported. These include, but are not limited to, extension of assay dynamic range, enhancement of assay selectivity, enablement of use of less reliable MRM transitions (e.g., loss of water) in MS/MS detection for better sensitivity, and coverage of in-processing and post-preparation stabilities [5,6,23].

While SIL IS is generally superior to analog IS in tracking the analyte of interest, some important facts need to be taken into consideration in method development. First, the SIL IS should have sufficient mass difference relative to the analyte. An IS with molecular weight that is $1 \mathrm{Da}$ higher than the analyte for every $100 \mathrm{Da}$ of molecular weight is ideal for a minimized cross interference due to isotopic contribution [24]. This general recommendation is based on isotopic distribution of the entire molecule. Given the consideration that multiple reaction monitoring (MRM) of the analyte is often employed in bioanalytical assays, isotopic interference can be further mitigated by carefully selecting the labeled position and product ion in MRM [25]. Second, an SIL IS containing isotopes such as ${ }^{13} \mathrm{C}$ and ${ }^{15} \mathrm{~N}$ is expected to behave more closely to the unlabeled analyte than a ${ }^{2} \mathrm{H}$-labeled one. Several case studies have demonstrated that ${ }^{2} \mathrm{H}$-labeled IS has a slight shift in retention time as compared with the analyte. If the method is not optimal, this slight difference may be associated with suboptimal tracking [26-28]. Third, even with ${ }^{13} \mathrm{C}$ - and ${ }^{15} \mathrm{~N}$-labeled IS, optimal tracking is not guaranteed if the method is not free from the impact of non-uniform matrix effect and a non-linear response over the intended dynamic range [29].

It is often that SIL IS is not readily available, especially in early phase of the project(s). The high cost and long period needed for synthesis are the major obstacles. In these cases, analog ISs are often employed [30-32]. When selecting an analog IS, it is preferable that the candidate has the same key chemical structure and functionalities, a similar size, and similar $\log \mathrm{D}$ [33] as the analyte of interest [5]. This could help reduce the difference between the IS and analyte in ionization efficiency and extraction recovery. Moreover, the same key functional groups may help track the analyte in functional group-based sample extraction, for example, derivatization [34].

Searching for a suitable analog IS is not easy and, sometimes, time consuming. To effectively identify a good IS, several candidates from a compound library are tuned individually and prepared in a single IS working solution, in other words, cocktail [32]. In this evaluation, the performance of each IS candidate is evaluated by accuracy, precision, linearity, sensitivity, ion suppression and other factors. The authors' lab conducted a similar evaluation to quantify five in-house model compounds with an IS cocktail consisting of five commercial compounds that have been widely used as ISs. The best IS candidate was then selected for each analyte based on the regression coefficient. The selected IS was further evaluated by comparing incurred sample reanalysis results obtained with analog IS versus original values obtained with SIL IS [UNPUBLISHED DATA]. 
It is worth mentioning that the selection of an appropriate IS for protein therapeutics is more complex, considering the complexity of protein LC-MS bioanalysis workflows [35,36. Same as IS selection for small molecules, SIL protein is always the most desirable choice as it can maximally compensate for the variabilities derived from the many steps of the workflows, including immunoaffinity capture (if applied) and enzymatic digestion [37,38]. However, SIL protein needs to be generated in cell culture and may not be available timely in most cases unless the project is heading to pivotal stage of the program. Alternative ISs, such as SIL protein analog, SIL surrogate peptide or SIL peptide containing the same cleavable group as the protein of interest have been successfully adopted and reported [39-41].

\section{Best practices of IS employment/handling}

Use of a proper IS is not a guarantee for the robustness of the intended bioanalytical method if proper IS handling is not employed. In practice, the earlier an IS is incorporated in the bioanalytical process, the more reliable the assay results will be. Ideally, the IS should be added to samples in the very first step after samples are aliquoted. By doing so, the IS goes through all the sample processing steps with the analyte. Any variability that happens to analyte during these steps is expected to be tracked and normalized by the IS.

The importance of IS addition as early as possible becomes more obvious in protein LC-MS bioanalysis, in which proteins are typically processed via enzymatic digestion followed by quantitation of surrogate peptide. An SIL protein analog that contains the same surrogate peptide sequence(s) as the protein generally outperforms an SIL surrogate peptide IS in tracking the variation during sample preparation [39]. This is because SIL surrogate peptide IS cannot compensate for the variability that occurs in extraction and digestion prior to LC-MS injection.

No matter at which stage an IS is incorporated into the bioanalysis workflow, thorough mixing of the IS and the analyte in sample matrix is essential. IS is generally prepared in a water-miscible solvent, such as acetonitrile or methanol or a combination of water and a water-miscible solvent. Upon addition of the IS working solution to biological samples, a step of vortex-mixing is necessary to thoroughly mix the IS with the analyte in sample matrix before the next step. With protein precipitation workflow, IS may be prepared in organic solvent (e.g. acetonitrile, methanol) and used directly for protein precipitation. If mixing is not thorough, inconsistent extraction recovery might be encountered for the analyte although SIL IS response may be consistent. This inconsistent extraction recovery is possibly due to incomplete release of the analyte, especially those highly protein-bound, from endogenous proteins during extraction. An efficient approach to minimize the recovery variability is to add a small volume/percentage of acid (e.g., formic acid, acetic acid) or base (e.g., ammonium hydroxide) into the protein precipitation solution or to pretreat the sample with a small volume of the acid or base to dissociate the analyte from proteins before protein precipitation. A small percentage of methanol in acetonitrile as protein precipitation solution was also effective in reducing variability in extraction recovery in protein precipitation. According to the authors' observation, in contrast from the solid pellet type of precipitates formed upon addition of $100 \%$ acetonitrile, the precipitates formed after addition of 10:90 $(v / v)$ methanol/acetonitrile were loose as floc. The assumption is that the protein-bound analyte in the floc precipitate will have more contact with the organic solvent, thus be much easier to be extracted during the vortex-mixing [Unpublished Data].

There is no strict rule on how much IS should be added to each sample as the reported amount of IS added ranges from a low percentage (\%) of the upper limit of quantification (ULOQ) [42] to a level higher than the ULOQ [43]. There is no health authority recommendation on IS concentration. In practice, the amount of IS that can be added to sample is dictated by assay selectivity/specificity. In this assessment, in addition to evaluating double blanks for the absence of analyte or IS response, possible contribution, if any, from the IS to the response of the analyte should be less than $20 \%$ of that observed for the analyte at the LLOQ level and the contribution of the analyte at the ULOQ level to the IS response should be less than 5\% of the IS response at the working concentration level [18]. Upon meeting the above criteria, IS concentration is recommended to be high enough to reduce systematic error in the analysis of unknown samples [44]. Otherwise, cross contribution from the analyte to IS due to chemical impurities and/or isotopic interference will cause the calibration curve to be increasingly quadratic as the IS concentration decreases $[1,44]$; in contrast, cross interference from IS to the analyte will have an impact on the accuracy of the low end of the Cs and/or QCs.

The scenario with multiple analytes with one or more ISs is complicated, especially when the analytes are structurally related, such as parent and metabolite. Nonetheless, the principle to define IS concentration is the same as aforementioned criteria of interference check. Moreover, a high level of IS concentration is recommended when 
ionization competition between the analyte and its SIL IS becomes another concern for metabolite quantitation using the same IS [4].

Tan $e$ t al. suggested to use a relatively large volume of IS working solution (such as $200 \mu \mathrm{l}$ or more if possible), considering small volumes of addition by repeater are more prone to imprecision than large ones [10]. With many sample preparation methods, such as solid-phase extraction or liquid-liquid extraction, it is often not practical to add a large volume of IS working solution. Nonetheless, addition of an appropriate volume, e.g., $>50 \%$ of sample aliquot, of IS working solution allows for simple visual differentiation of sample wells with IS added from those with no IS added. In addition, double IS spiking can be also easily spotted visually.

\section{Evaluation of IS performance}

In general, the tracking ability of an IS might be directly judged from the linearity of the calibration curve and the accuracy and precision of QC results, the latter of which should meet the well-known acceptance criteria, i.e., $\leq \pm 15 \%$ bias ( $\pm 20 \%$ for the LLOQ) for accuracy and $\leq 15 \%$ CV ( $20 \%$ for the LLOQ) for precision. From IS point of view, a tighter bias\% and CV\% values for the Cs and QCs may be associated with a better tracking of the IS to the analyte, although other factors, for example, best practice on bench, might also play a role.

Matrix effect (signal suppression or enhancement) has been regarded one of the major sources of inaccurate bioanalytical results. A simple approach to evaluate matrix effect is the post-column infusion experiment. In this experiment, a constant flow of analyte or IS solution is introduced into the mobile phase post-column, which results in a raised and steady background MS signal. An extracted blank sample is then injected onto the LC-MS system. Any eluting components that interfere with the ionization of the analyte or IS will cause a disrupted (decreased or increased) background MS signal. A comparison of the retention time of the analyte and IS with the obtained background MS signal could help unveil the presence and, possibly, degree of matrix effect. If needed, chromatographic method and/or sample preparation method should be modified to ensure both the analyte and IS are free from matrix effect before method validation. During method validation, matrix effect should be systematically evaluated again by comparing response of the analyte and/or IS in post-extraction spiked blank matrix versus neat solution. Ratio of $<1$ suggests signal suppression and ratio of $>1$ indicates signal enhancement. IS is expected to experience similar matrix effect as the analyte. In the other words, the IS-normalized matrix factor (matrix effect of the analyte / matrix effect of the IS) should be close to one across the low, mid and high concentrations. More importantly, evaluation of a number of individual matrix lots is crucial to assess the possible lot-to-lot variation, which is predictive of assay suitability for analysis of study samples.

IS performance/tracking ability can also be assessed from selectivity and specificity, in particular, in the presence of hemolysis and hyperlipidaemia. QCs prepared in these special matrices should be assessed in method development and/or method validation. If the calculated analyte concentrations in those QCs are outside of $\pm 15 \%$ of nominal values, method optimization is necessary. It is worth noting that the timing of IS addition is essential during the stability assessment. Since it has the same chemical structure as the analyte, the SIL IS could undergo the same degradation as the analyte, thus yielding to misleading stability results if instability has not been addressed.

\section{Common root causes of abnormal IS response variability}

Given the complexity of biological matrices, multiple steps of sample processing for any given extraction method, and possible variation in injection volume, it is not uncommon to see variable IS response throughout a bioanalytical run. This variability might be higher in sample analysis runs than in method validation runs, considering the difference in matrix composition between the blank matrices used for preparing Cs/QCs and possible subject-tosubject variation of incurred samples. This phenomenon is more or less anticipated and considered acceptable as long as IS tracks the analyte and IS response variability is not notably high. Notable variation in IS response could be sporadic or systematic. Sporadic variation is often evidenced by one or more samples from the same subject(s) showing unexpectedly high or low IS response when compared with the rest samples of the same subject(s) or the $\mathrm{Cs} / \mathrm{QCs}$ in the same run. In contrast, systematic variation is characterized by a directional trending of either high or low IS response of subject samples in a batch compared with the prepared samples (calibration standards and QCs) within the same run.

Many common root causes have been reported that, alone or together, may contribute to abnormal IS response variability in LC-MS bioanalysis. These causes include, but are not limited to, the following: study subject-specific matrix effect and interferences, instrument/device-related issues, ionization competition between analyte and its 
Table 1. Cases of abnormal internal standard response variability with negative impact that led to re-development of the method.

\begin{tabular}{|c|c|c|c|c|c|}
\hline Root cause & IS type & IS response pattern & Trouble shooting & Remediation & Ref. \\
\hline Interference & SIL IS & $\begin{array}{l}\text { IS responses in the undiluted } \\
\text { samples were much higher than } \\
\text { responses from the diluted } \\
\text { samples }\end{array}$ & $\begin{array}{l}\text { A co-eluting interfering peak was present around the } \\
\text { retention time of IS in the plasma from subjects with } \\
\text { impaired liver function }\end{array}$ & $\begin{array}{l}\text { The assay was redeveloped to } \\
\text { separate the IS from the } \\
\text { interfering component(s) }\end{array}$ & [3] \\
\hline Matrix effect & Analogue & $\begin{array}{l}\text { The IS response in the study } \\
\text { samples was systematically } \\
\text { higher than those of Cs and QCs }\end{array}$ & $\begin{array}{l}\text { More lots of blank with IS only were tested and the } \\
\text { respective study samples were diluted with pre-tested } \\
\text { blank plasma to confirm the observations }\end{array}$ & $\begin{array}{l}\text { All study samples were } \\
\text { reanalyzed after a 5-fold dilution } \\
\text { with the same blank plasma used } \\
\text { for } \mathrm{Cs} \text { and QCs }\end{array}$ & [3] \\
\hline Interference & Analogue & $\begin{array}{l}\text { IS responses in post-dose urine } \\
\text { samples were about } 5 \text {-fold } \\
\text { higher than those of Cs }\end{array}$ & $\begin{array}{l}\text { The chromatographic condition was modified to } \\
\text { resolve the analyte and IS. A number of early eluting } \\
\text { peaks were observed in the channel used to monitor } \\
\text { the IS }\end{array}$ & Use the revised condition & [13] \\
\hline Matrix effect & Not known & $\begin{array}{l}\text { IS responses from incurred } \\
\text { samples were more than 3-fold } \\
\text { higher than those of Cs and QCs }\end{array}$ & $\begin{array}{l}\text { Selected incurred samples were diluted with control } \\
\text { matrix. Comparable IS responses were observed } \\
\text { between incurred samples and Cs/QCs; however, } \\
\text { analyte concentrations in the diluted incurred } \\
\text { samples were not consistent with those of the } \\
\text { undiluted incurred samples }\end{array}$ & $\begin{array}{l}\text { More effort is needed for the } \\
\text { method }\end{array}$ & [8] \\
\hline Instability & SIL IS & $\begin{array}{l}\text { In an enzyme activity assay, an } \\
\text { increasing IS response was } \\
\text { observed with increasing analyte } \\
\text { concentration in stored sample } \\
\text { extracts }\end{array}$ & $\begin{array}{l}\text { The analyte (labeled enzyme product) and IS react } \\
\text { over time with the thiol substrate. Competition from } \\
\text { higher concentrations of the analyte result in less } \\
\text { chance for the reaction of the IS }\end{array}$ & $\begin{array}{l}\text { Reduce reaction by addition of } \\
\text { concentrated unlabeled enzyme } \\
\text { product to stored processed } \\
\text { samples }\end{array}$ & [8] \\
\hline Instability & SIL IS & $\begin{array}{l}\text { A reduced IS response was } \\
\text { observed in post-dose samples }\end{array}$ & $\begin{array}{l}\text { The stable-labeled IS functioned as an alternative } \\
\text { substrate for the enzyme. The degradation of the IS } \\
\text { alone resulted in the rejection of the analytical run } \\
\text { data, but the analyte peak response would have also } \\
\text { been reduced after sample thawing, even on wet ice } \\
\text { and any measured concentrations would have been } \\
\text { meaningless }\end{array}$ & $\begin{array}{l}\text { The IS response data and } \\
\text { subsequent investigation lead to } \\
\text { revalidation of the method using } \\
\text { added stabilizer for the matrix }\end{array}$ & [8] \\
\hline $\begin{array}{l}\text { Instrument } \\
\text { malfunction }\end{array}$ & Not known & $\begin{array}{l}\text { The IS response was } \\
\text { systematically lower every } 11 \text { and } \\
12 \text { samples in the run. The } \\
\text { variation was insufficient to } \\
\text { trigger a re-analysis }\end{array}$ & $\begin{array}{l}\text { Malfunction of the instrument employed to } \\
\text { distribute the IS }\end{array}$ & $\begin{array}{l}\text { All runs in the study were } \\
\text { affected, as well as potentially } \\
\text { other studies }\end{array}$ & [7] \\
\hline $\begin{array}{l}\text { Unknown } \\
\text { (spiking error or } \\
\text { others) }\end{array}$ & Analog & $\begin{array}{l}\text { A gradual decrease in the IS } \\
\text { response at the end of the run. } \\
\text { The variation was within } \pm 50 \% \\
\text { criteria and run was acceptable }\end{array}$ & $\begin{array}{l}\text { The last four QC samples failed with high results. The } \\
\text { last three ISR samples also failed as concentrations } \\
\text { being more than } 20 \% \text { higher than the initial } \\
\text { determination }\end{array}$ & $\begin{array}{l}\text { Recommended to reconsider the } \\
\text { choice of IS. It was deemed likely } \\
\text { that other runs were affected for } \\
\text { this study }\end{array}$ & [7] \\
\hline
\end{tabular}

SIL IS, spiking errors and instability. These cases were well captured in the recent special issue on IS response monitoring and other early publications $[3,7-10,12,13,27,45,46]$.

Summarized in Tables 1 and 2 are some representative cases of abnormal IS response variability and associated assessment and/or remediation. Listed in Table 1 are cases where the abnormal IS response variability unveiled method defects that should have been discovered and/or addressed properly during method validation. Method optimization or even redevelopment of the method was needed. In contrast, investigation into some other cases of abnormal IS response variability showed that the IS tracked the analyte very well, although the observed variation was apparent for some samples (Table 2). Remediation of the assay is not necessary upon adequate scientific assessment.

\section{Subject-specific matrix effect \& interference}

Subject-specific matrix effect (i.e., signal suppression or enhancement) and interference are interchangeably the two lead causes of abnormal IS response variability in LC-MS bioanalysis. Although assay specificity/selectivity should have been assessed thoroughly during method development and/or validation according to the current health authority guidelines, common industry practice and applicable SOPs, it should be recognized that the components in study samples are much more complex than the blank matrices used for preparation of Cs/QCs. This complexity can be associated with subject-specific endogenous components due to different disease state or 
Table 2. Cases of abnormal internal standard response variability and investigation confirmed the original values.

\begin{tabular}{|c|c|c|}
\hline Root cause & IS type & IS response pattern \\
\hline $\begin{array}{l}\text { Instrument } \\
\text { malfunction }\end{array}$ & Not known & $\begin{array}{l}\text { A random drop in IS response up } \\
\text { to 2-fold in several samples in the } \\
\text { later part of the run. One QC } \\
\text { next to these study samples was } \\
\text { affected }\end{array}$ \\
\hline $\begin{array}{l}\text { Matrix-induced } \\
\text { degradation }\end{array}$ & SIL IS & $\begin{array}{l}\text { An extreme variability (decreased } \\
\text { IS response) was observed in } \\
\text { samples from malaria patients }\end{array}$ \\
\hline Matrix effect & SIL IS & $\begin{array}{l}22 \text { out of the } 158 \text { incurred } \\
\text { samples exhibited a more than } \\
2 \text {-fold increase in IS response }\end{array}$ \\
\hline
\end{tabular}

Instrument Not known A random drop in IS response up Re-inject the run on a different LC-MS system or on

The injected analyte and IS was

$[10,12]$

the same system after the needle being cleaned

reduced to exactly the same

extent, so the obtained results

were not affected

Plasma samples from malaria patients contain varying Since analyte and IS are affected

levels of iron, which resulted in degradation of

analyte and IS during protein precipitation step. Replacing the protein precipitation step by a solvent-free solid-phase extraction largely reduced the variability of the IS response

to the same extent, the results

were still reliable. However

$\sim 15 \%$ of original results were unquantifiable due to complete

loss of response. Improved method was needed

Endogenous bile acid, TCA had overlapping retention Injection of different volumes of time as the analyte. No significant IS response enhancement was observed in the case of complete separation between analyte and TCA samples with normal IS response or enhanced IS response gave unchanged response ratio between analyte vs IS. Therefore all results were accepted and no samples were re-analyzed

$\begin{array}{lll}\begin{array}{l}\text { Inhomogeneity } \\ \text { in stored } \\ \text { extracted } \\ \text { sample }\end{array} & \text { SIL IS } & \begin{array}{l}\text { Upon reinjection of an extracted } \\ \text { batch after } 120 \mathrm{~h} \text { storage in the } \\ \text { autosampler, an increase in IS } \\ \text { response variation was observed }\end{array} \\ \text { Matrix effect } & \text { SIL IS } & \begin{array}{l}\text { The IS responses of parent drug } \\ \text { and metabolite were higher in } \\ \text { study samples than those in Cs } \\ \text { and QCS }\end{array}\end{array}$

IS responses increased and decreased across the run. All validation results passed, which suggests that the IS was affected to the same extent as the analyte

No method modifications needed

Repeat analysis after a 10-fold dilution with the matrix used to prepare Cs/QCs confirmed original results. IS response in diluted sample was comparable with those of Cs/QCs

QCs prepared with pre-dose samples with enhanced IS response met the acceptance criteria

Matrix effect SIL IS $\quad$ In clinical studies, incurred
samples showed consistent IS
response. However, in some runs,
IS response in CS/QCs was about
half of the incurred samples

Two matrix lots used to prepare Cs/QCs had different The IS compensates for the matrix effect. Selected incurred samples diluted with either matrix lot met the ISR acceptance criteria variation of the analyte in matrix with ion suppression. No action needed

Matrix effect has no impact to modification was needed

No impact to the results as long as signal/noise ratios were high enough

malfunction
decrease gradually slowed down

Instrument Not known Sudden drops of IS response

malfunction were observed during the run. Accuracy of Cs/QCs with abnormal IS response was not affected

\begin{tabular}{lll}
\hline $\begin{array}{l}\text { Mixed use of } \\
\text { different caps } \\
\text { during LLE }\end{array}$ & SIL IS & $\begin{array}{l}\text { IS responses in most of extracted } \\
\text { samples (Cs, QCs and study } \\
\text { samples) were much lower than } \\
\text { the expected }\end{array}$ \\
\hline Matrix effect & SIL IS & $\begin{array}{l}\text { Samples from a few subjects } \\
\text { showed consistently higher IS } \\
\text { response than the others }\end{array}$ \\
\hline Matrix effect & SIL IS & $\begin{array}{l}\text { IS response of incurred samples } \\
\text { was approximately half of that of } \\
\text { CS/QCs }\end{array}$
\end{tabular}

The needle of auto-sampler was partially or completely blocked after piercing the caps or cover

No impact on accuracy as long as sufficient sensitivity can be obtained

The mass spectrometer got 'charged' due to

Rubber cap absorbed some analyte and IS in organic No impact solvent, thus reduced recovery. Issue was resolved after switching to PTFE-lined caps

Matrix effect of blank matrix used for Cs/QCs preparation and the pre-dose sample from the subjects with high IS response were compared by post-column infusion. Ion suppression in blank matrix was proved to be the root cause

Samples were reanalyzed with varying degrees of

Results from the diluted samples

No impact

dilution down to the point where the IS response of the diluted samples was similar to those of Cs and QCs

were similar to the original samples, which demonstrated no impact

All the study samples were hemolyzed. Analyte was

No impact spiked to the control group samples and hemolyzed blank plasma ( 1 and $2 \%$ hemolysis). The results showed suppressed IS response, but accurate concentration samples was much lower than that of Cs/QCs

\begin{tabular}{|c|c|c|}
\hline $\begin{array}{l}\text { Divergent } \\
\text { trends with } \\
\text { unidentified } \\
\text { cause }\end{array}$ & Not known & $\begin{array}{l}\text { A gradual increase in IS response } \\
\text { was seen in incurred samples, } \\
\text { which contrasted with a gradual } \\
\text { decrease in the IS response in QC } \\
\text { samples }\end{array}$ \\
\hline
\end{tabular}

The repeat run did not show any trend or systematic No impact difference and the repeat analysis confirmed the concentration measured initially

C: Calibration standard; IS: Internal standard; ISR: Incurred sample reanalysis; QC: Quality control; SIL IS: Stable-isotope labeled internal standard; TCA: Taurocholic acid. 
diet, drug metabolite(s), co-medication and its metabolite(s), improper sample processing that results in different degrees of hemolysis, and dosing vehicle, among others. [18,47].

Cases of study subject-specific interference have been reported for abnormal IS response variability due to a co-eluting compound [13] or due to non-resolved peaks contributed from endogenous components, metabolites or co-medications with a 'validated assay' [3,13]. In a case study reported by Woolf et al. [13], one of the over-the-counter (OTC) drugs was found to have the same molecular weight as the SIL IS of the analyte of interest. This OTC shares the same MS channel as the SIL IS, and unfortunately, it was not fully resolved from the SIL IS initially. If this had not been caught for modification of the chromatographic method, the exposure of the analyte of interest would have been under-estimated due to the elevated IS response from the OTC contribution [13].

In a separate case study, Olsson et al. reported that unexpectedly high SIL IS signal was observed for $14 \%$ of plasma samples in a 3-month toxicity study, while no such matrix effect was observed during method development and validation [48]. The presence of an endogenous bile acid namely taurocholic acid (TCA) was identified to cause signal suppression of the IS as the acid had overlapping retention time as the IS. Further investigation unveiled that the level of bile acids in plasma was closely related to food intake or certain diseases. This may explain why the matrix effect was not detected in method validation but in sample analysis [44].

Fung et al. designed a series of experiments to evaluate the impact of hemolysis on IS response using two model compounds and their corresponding SIL ISs [22]. The results demonstrated that an increase in hemolysis level (prepared by mixing plasma with different amounts of lysed whole blood from 0 to $50 \%$ in volume) was associated with a decrease in IS response, as well as less accurate and precise QC results. Dilution of the hemolyzed QCs with a higher dilution factor was associated with a measured QC result being closer to the nominal values. Based on this observation, Fung et al. proposed to use the IS response as a universal tool to assess the possible effect of hemolysis and identify study samples for repeat analysis after dilution. The same strategy can be adopted for the analysis of hyperlipemic samples or any other type of variable matrix effect.

Dosing vehicle is another source of ion suppression, which is often an issue in preclinical studies where solubilizers, such as polyethylene glycol (PEG) or polysorbate 80 (Tween 80 ) are often employed in formulation [49,50]. These vehicles may cause significant signal suppression for the analyte and/or IS if appropriate sample cleanup or chromatographic separation is not in place, especially for samples collected at early time points (excluding pre-dose samples) of iv studies.

\section{Instrument/device related issues}

In sample extraction, a liquid handler, such as TomTec and Tecan, is employed for transfer of non-critical solutions (e.g., buffer, wash solution, eluting solution, reconstitution solution) or sample extracts (e.g., supernatant). Although liquid handlers are routinely calibrated, unexpected issues, e.g., seal defects, often lead to less volume being transferred. Consequently, the respective samples have IS response lower than the average. Similarly, partial blockage of the syringe in the autosampler when piercing through the sample plate cover/seal is associated with less and inconsistent volumes injected onto LC-MS/MS, leading to highly variable IS responses across the run. Nonetheless, IS response variability caused by inaccurate transfer of non-critical solutions or partial blockage of the needle has no impact to the final measurement since analyte/IS ratio is constant throughout the entire process as long as the sensitivity is sufficient.

An LC method with insufficient elution of matrix components after each injection cycle may lead to accumulation of matrix components on column. Release of those accumulated matrix components can result in progressively increased matrix effect to the analyte and IS, leading to inconsistent IS response due to signal suppression or enhancement as a result of matrix effect.

Mass spectrometer should be fully equilibrated prior to start of a run sequence. System suitability samples are commonly injected to monitor the readiness of the instrument. The number of injections needed is compound and/or instrument dependent. In some cases, many injections are made until a stable analyte and IS response pattern is observed. Failure to adequately equilibrate the instrument is often evidenced by inconsistent IS response across the run. 'Charging' of the mass spectrometer might occur when the rods of quadrupoles are contaminated, especially when high flow rate is used without split [10]. Consequently, the number of ions transmitted will gradually decrease in a similar way as the charging of a capacitor. This phenomenon is evidenced by a continuously decreased IS response during a run. Whether or not the IS still tracks the analyte is uncertain and may be compound dependent. 
Ionization competition between analyte \& its SIL IS

In some cases, the response of SIL ISs are suppressed with the increasing concentration of the corresponding analyte in LC-ESI-MS [51,52]. This phenomenon is caused by the competition among ions for the limited number of excess charged surface sites on droplets in gas phase during the ESI process [4]. The ionization competition between the analyte and its SIL IS has minimal or no impact on the slope of the calibration curve and quantitation of the target analyte, because the response factor (analyte concentration normalized response ratio of analyte vs SIL IS) is constant [52]. However, in the case of metabolite quantification using the parent SIL IS (considered as analogue IS), if the SIL IS is subjected to ion suppression by the parent analyte, the increased metabolite/IS response ratio due to such an ion suppression will result in over-estimation of the metabolite concentration. In Jian's report with propranolol and its metabolite 4-hydroxypropranolol as model compounds, significant bias for metabolite quantitation was due to the high propranolol concentration that suppressed D7-propranolol ionization [4].

Common approaches to mitigate the impact due to ionization competition include but are not limited to switching ionization mode from ESI to APCI [53]; increasing IS concentration; selection of other analog IS for metabolites, or dilution of samples that contain a high concentration of parent analyte, etc.

\section{Spiking error}

Addition of IS working solution at a fixed volume to all the appropriate samples is a key to the accurate and precise quantitation of a bioanalytical run. Any error in the amount of IS added to a sample will have significant impact to the measured concentration. It is not rare to see sporadic variation (very high or very low) in IS response during a run. A sample with IS response approximately 2 -fold higher than the average value often suggests double spiking of the IS working solution. In contrast, a sample with very low IS response might be caused by missed or partial spiking of the IS working solution due to bubble in repeater pipet or improper use of repeater. All of above issues are caused by human error and should be avoided by building up good practice in sample preparation.

\section{Instability}

Stability assessment is one of the major activities in method development and validation. In theory, there should be no stability issues for both the analyte and IS with a well-developed and fully validated bioanalytical assay. Abnormal IS response due to the issue of instability has not been frequently reported, except for enzyme-involved assays. Buonarati et al., reported a case study with inconsistent IS response due to instability in an enzyme activity assay. The enzyme product analyte and its SIL IS were found to react over time with the thiol substrate and compete with each other. This led to an increased IS response with increasing enzyme product analyte concentration in runs stored overnight [8]. In a second case study reported by Buonarati et al., biomarker was monitored with administration of enzyme therapeutic designed to reduce the endogenous biomarker [8]. Both the analyte and SIL IS were found to degrade in the presence of the enzyme therapeutic at $4^{\circ} \mathrm{C}$, which would have made the obtained data meaningless unless the method was re-validated using a stabilizer.

Another interesting case relevant to compound instability was reported by Lindegardh et al. [46]. An LC-MS/MS assay with protein precipitation was successfully established to quantify artesunate (ARS), an anti-malarial drug, and its metabolite dihydroartemisinin (DHA) in human plasma. Excellent assay performance was demonstrated in the method validation in support of clinical study. However, a huge IS response variability was observed for the study samples collected from the malaria patients. Investigation into this observation revealed that the organic solvent used in sample treatment had caused the release of ARS and DHA from protein binding and the released ARS and DHA reacted with iron (II)-heme complex present in the malarial patient samples. This matrix-induced instability was resolved by replacing protein precipitation with solvent (organic)-free solid phase extraction.

\section{Identification of abnormal IS response variability \& assessment of its impact on LC-MS bioanalysis}

In reality, not all the abnormal IS responses are associated with erroneous measurements in LC-MS bioanalysis. For example, as long as the IS is thoroughly mixed with the analyte in all samples (Cs, QCs and unknowns) in the very beginning of the extraction process, the abnormal IS response due to inconsistent injection volume, inaccurate addition of reconstitution solution or inaccurate sample extract transfer, etc., generally have no impact on the measured results. In contrast, the abnormal IS response variability due to subject sample specific matrix effect and/or interference, error in IS working solution addition, or instability, etc., may have an impact on the accuracy of the measurement. A proper process should be in place to guide identification of unexpected IS response 
variation, sporadic or systematic, and investigation into its root cause followed by assessment of possible impact. Such an investigation and impact assessment should lead to the needed action to confirm and report the obtained results or modify/redevelop the assay method.

\section{Identification of abnormal IS response variability}

A simple approach to identify abnormal IS response variability is to compare the IS response of interest versus the mean, which could be the average of all the knowns (Cs and QCs) and unknowns except double blanks; unknowns only; or knowns (Cs and QCs) only. A recent survey unveiled that the acceptance window for the IS response variability varies from one organization to the other, ranging from $25-175 \%, 30-170 \%, 50-150 \%, 50-175 \%$, to 50-200\%, etc. Some laboratories set tighter criteria for analog IS than SIL one while others do not differentiate them. Any samples with IS response outside of the predefined acceptance window need to be reanalyzed in singlet or duplicate for confirmation.

While the above approach is straightforward and easy to follow, the acceptance windows are more or less arbitrary with not much sound scientific or regulatory basis. There has been a concern that this simple approach may lead to inaccurate measurements due to suboptimal IS tracking although the respective IS response(s) may fall in the predefined window. Examples have been reported that IS responses with variability within 50 to $150 \%$ range actually did not always reflect the correct measurement [7]. However, too narrow the acceptance criteria is impractical given the fact that IS always carries a certain degree of variation $[7,8,12]$.

Several procedures have been recommended to facilitate a deep understanding of IS variability from scientific perspective $[5,7,9,11-13]$. Those recommendations can be summarized into the following two major areas: classify the abnormal IS response variability into sporadic and systematic ones; investigate whether or not the IS response variability in Cs/QCs have the similar trend versus respective study samples. A decision tree was proposed by van de Merbel et al. to help identify the root cause of unexpected IS response variability in LC-MS bioanalysis [12]. Other than evaluating IS response in Cs/QCs and study samples, a comparison of trend in IS response variability between Cs/QCs and samples should also be focused.

Herein, the authors proposed a different decision tree for a systematic investigation of abnormal IS response variability for each sample analysis run. As depicted in Figure 1, the very first step of the decision tree is to plot the IS response versus injection sequence number to evaluate the consistency of IS response across the entire run. Any obvious outliers should be identified from this step. Following respective SOP, the mean IS response and \%Bias of IS response of study samples versus the mean are calculated. Any samples with IS response falling outside of the acceptance criteria (mean $\pm \mathrm{x} \%$ ) are flagged for repeat analysis following the remaining route of the decision tree. The root causes of the IS response outlier(s) could be human error, method related or sample matrix specific. Repeat analysis of the respective samples is effective in identifying any spiking errors or instrumentation issues in the original run. However, if the abnormal IS response variability is due to study sample-specific matrix effect and/or interference, a simple repeat reanalysis might not be effective in identifying the hidden root cause. Both repeat and initial results might not be representative of the true values. Thus, it is highly recommended to conduct the repeat analysis with a dilution factor different from the initial analysis using the same blank matrix as for the preparation of the Cs and QCs, if possible. In this repeat analysis, the IS response in the diluted study samples is expected to be similar to those of Cs and QCs. If the repeat value matches with the original result (within $\pm 20 \%$ ), the original value should be accepted and reported. Depending on lab resources and/or study timelines, further investigation may continue, but is not mandatory. In contrast, if different results are obtained from repeat analysis with comparable IS response between Cs/QCs and study samples, a closer look into the IS response pattern of the respective samples and Cs/QCs in both the initial and repeat analysis is necessary. A similar IS response between samples and Cs/QCs in the repeat run while different in the initial run may suggest a possible spiking error or matrix effect in the initial analysis. Under this circumstance, the repeated result should be reported. Otherwise, investigation should continue if abnormal IS response still exists in the repeated sample.

It has been aforementioned that IS response falling within the SOP pre-defined acceptance window does not guarantee that the obtained results are accurate and free from issues. Instead, in regulated LC-MS bioanalysis, necessary efforts should be made to evaluate IS response pattern/trend of the unknowns versus the knowns (Cs and QCs) to identify systematic IS response variability that might impact the accurate measurement of the respective study samples. With this regard, it is worth noting that spreading QCs properly across the entire run and/or bracketing a group of study samples with proper QCs is expected to help this exercise. In this exercise, if the abnormal IS response variability is only observed in study samples, but not in Cs/QCs, the abnormal IS response 


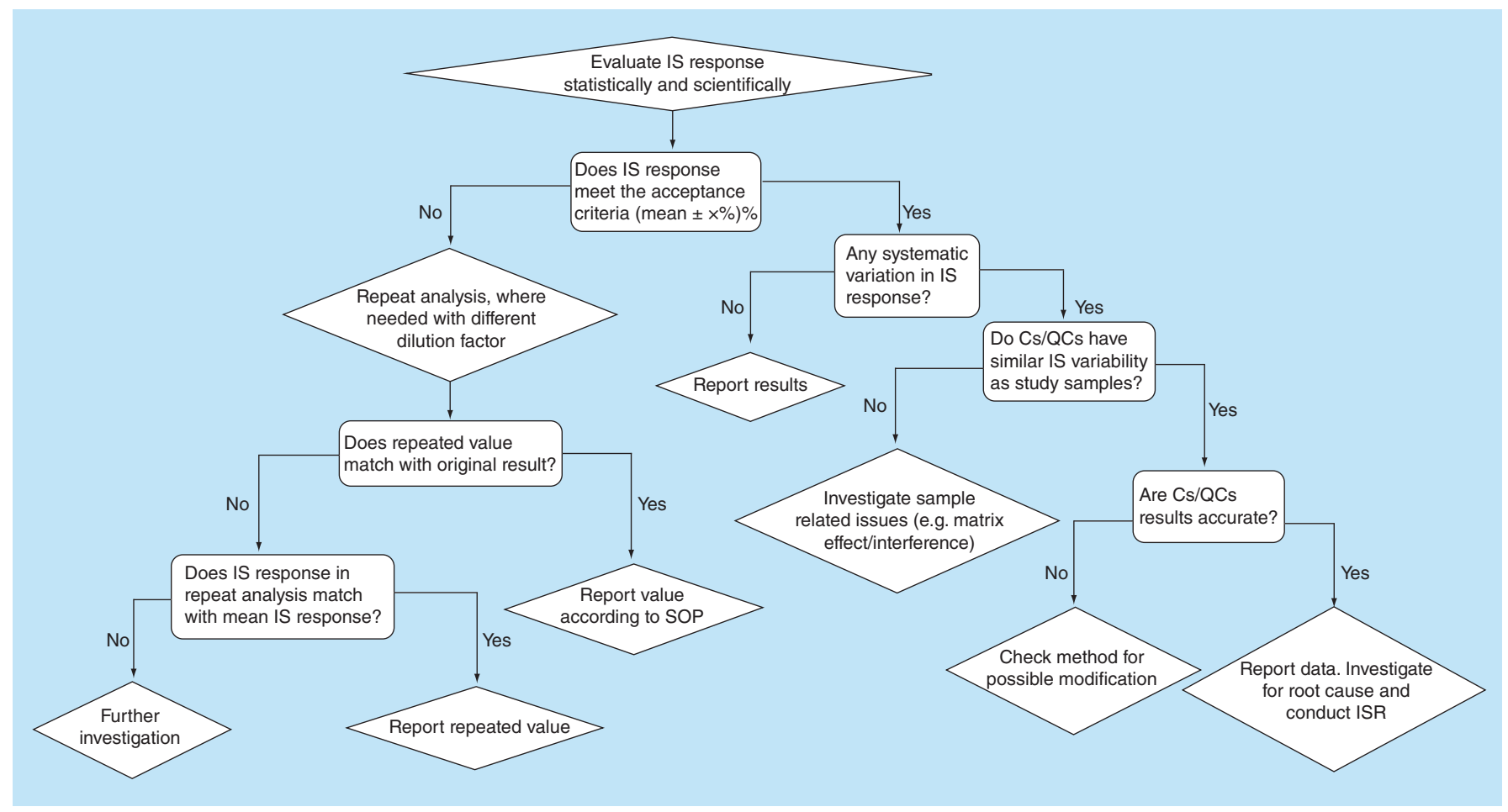

Figure 1. Decision tree to investigate abnormal internal standard response variability of individual samples from each sample analysis run.

is most likely to be due to sample related issues, such as matrix effect, interference, etc. Grouping respective study samples by subjects and/or TK/PK profiles is preferred in this trend analysis. For example, a consistent high or low IS response (versus Cs and QCs) in the respective subject(s) samples may suggest matrix-induced signal enhancement or suppression for the respective subjects. Time-dependent IS response variability in the post-dosed TK/PK profile samples may suggest matrix effect introduced by dosing vehicle or interference due to co-medication. The impact due to these root causes needs to be further assessed with approaches described in the section below.

In general, IS responses in study samples are expected to be comparable to those in Cs/QCs if the method is robust and sample processing is consistent. In the case where abnormal IS response variability was observed for both the study samples and Cs/QCs, the root cause may have more to do with sample processing and or instrument/device. Analysis of IS response versus injection sequence is helpful to reveal the root cause. For instance, a gradually decreasing IS response may suggest an issue of MS charging; a repeated occurrence of lower IS response than expected may suggest blockage or leaking of the systems, e.g., Tecan or Tomtec. In this case, examination of Cs/QC result(s) might help with understanding whether the abnormal IS response variability is an issue. If the Cs/QCs that bracket the respective samples did not meet the acceptance criteria, the observed IS response variability may negatively affect respective sample results. In this case, each step of sample processing needs to be investigated. In contrast, if all Cs/QC sample results meet the acceptance criteria, then the observed abnormal IS response variability may have been associated with non-impactful errors such as inconsistency in non-critical solvent addition, sample extract transfer, and/or inconsistent injection volumes, etc. Nevertheless, investigation into the root cause is still necessary to prevent recurrence. It is also recommended to include some respective samples in incurred sample reanalysis (ISR) for confirming the no-impact. The passing ISR results may serve as additional supporting evidence of the reported values.

\section{Assessment of possible impact due to abnormal IS response variability}

As summarized in Table 1 and Table 2, the same root cause of abnormal IS response variability might be associated with negative impact (Table 1) or no impact (Table 2) on the measured results, depending on how well the IS tracks the analyte of interest in the process of sample preparation and LC-MS. With this regard, proper assessment has to be carried out before making such an impact or no impact judgement. Whenever a no impact is concluded from the 
assessment, some respective samples should be selected for ISR to generate additional supporting data. In contrast, whenever a negative impact is unveiled from the assessment, the method has to be modified/optimized, which is followed by partial validation or re-validation before reanalysis of affected samples or the entire study sample sets. Recommended below are some practical approaches for the assessment of possible impact due to abnormal IS response variability. Among these approaches, simple repeat analysis is ideal for troubleshooting IS response variability due to human error or device malfunction. In contrast, the rest of three approaches are good fit for the issues due to subject sample specific matrix effect/interference.

- Simple repeat analysis: If spiking error in the step of IS addition is suspected to be the root cause of the abnormal IS response variability, it can be easily identified and/or confirmed by re-assaying the same sample(s) and evaluating whether the IS response is back to normal. For the abnormal IS response variability caused by malfunction of equipment/device, such as liquid handler and autosampler, etc., samples should be re-assayed/re-injected after the issue is fixed, or using different equipment. The difference (\%) between the repeated values and the original ones should be evaluated to determine whether or not the abnormal IS response variability has any impact to the measurement of the analyte.

- Analysis of QCs prepared using pre-dose or baseline samples: As discussed above, subject specific-matrix effect (i.e., signal suppression or enhancement) and interference are interchangeably the two lead causes of the abnormal IS response variability in LC-MS bioanalysis. In both preclinical and clinical studies, pre-dose or baseline samples are often collected. Although these samples have the same matrix composition as the dosed samples with abnormal IS response variability as they are basically collected from the same respective subjects, they generally do not contain the analyte(s) of interest and/or its metabolite(s). In assessing the possible impact due to abnormal IS response variability, these pre-dose or baseline samples should be used to prepare QCs (low and high, or low, mid and high) and assayed along with regular Cs and QCs. It is worth mentioning that this approach is a better fit for assessing the possible impact due to endogenous components induced abnormal IS response variability as metabolite(s) or drug formulation related materials are not present in those samples.

- If the observed IS responses in these QCs are comparable with those obtained from the respective study samples and the measured analyte concentrations are close (bias within $\pm 15 \%$ ) to the nominal values, a conclusion can be drawn that the abnormal IS response variability has no impact on the analysis of the respective study samples.

- If the observed IS responses in these QCs are comparable with those obtained from the respective study samples but the measured analyte concentrations are outside $\pm 15 \%$ (bias) of the nominal values, further investigation into the method is warranted.

- If the observed IS responses in these QCs are different from those obtained from the respective study samples, the root cause of the abnormal IS response variability might have been due to non-sample related issues.

- Parallelism: For the abnormal IS response variability due to matrix effect and/or interference, simple repeat analysis of respective samples without any change most probably will give measured values and IS response pattern close to the original runs. Under this situation, the difference/similarity (\%) between repeated results and the original data is unlikely to be supportive for any firm assessment of impact due to the abnormal IS response variability. In this case, in addition to the approach of preparing QCs using pre-dose or baseline samples discussed above, an approach called 'parallelism' should be considered and employed. In this approach, samples with abnormal IS responses are diluted (5-fold and above) with control matrix that is used to prepare Cs/QCs prior to aliquoting for extraction. The diluted samples are assayed along with regular Cs and QCs. An apparent limitation of this approach is the analyte concentration levels in the samples. The analyte concentration in the samples should be high enough so that they are still measurable after large scale of dilution.

- If the IS responses in the diluted samples are similar to those of Cs/QCs and the repeated results confirm the correctness of original values (within $\pm 20 \%$ ), it can be concluded that the IS tracks the analyte well in the respective subject samples and the abnormal IS responses do not have impact on the quantitation.

- If the IS responses in the diluted samples are different from those of Cs/QCs, additional repeat analysis should be conducted with a higher dilution factor until the observed IS responses being similar to those of 
Cs/QCs. If the repeated results confirm the correctness of original values (within $\pm 20 \%$ ), the abnormal IS responses can be judged to have no impact on the quantitation of the respective samples.

- If the IS responses in the diluted study samples are similar to those of Cs/QCs but the repeated values do not match (outside $\pm 20 \%$ ) with the original ones, further investigation into method or lab operation is necessary. This investigation might lead to modification or re-development of the intended method.

Standard addition: In the case where the analyte concentrations in the affected study samples are at the low end of the calibration curve and dilution for reanalysis as discussed above is not an option, standard addition might be considered for assessing the possible impact due to the abnormal IS response variability [13]. In this approach, different amounts of analyte are added into the study samples, followed by the addition of IS at a constant concentration. A straight line with linear regression is expected when plotting the response of the analyte versus standard added. The concentration of the analyte in study sample can be calculated from the point $(x$-axis) at which the extrapolated linear regression line crosses the concentration axis at zero signal. Scientifically, this approach may be reasonable. However, establishing individual internal calibration curves for each sample is obviously time consuming in data processing and limited to available sample volume. The applicability of this approach may not be realistic when supporting study with large amount of samples.

\section{Future perspective}

IS plays an important role in LC-MS bioanalysis by compensating for the variability of the analyte of interest in sample extraction and LC-MS. The trackability of IS is largely dependent on the closeness of its physicochemical properties to the analyte of interest and appropriate employment of IS in sample extraction. A general recommendation is to incorporate the IS in sample processing procedure as early as possible and thoroughly mix IS with the analyte in biological samples prior to extraction to ensure maximum compensation for the variations of the analyte of interest [7]. In addition, selection of IS concentration and volume to be added can have impact on assay performance as well. One should recognize that incurred samples are much more complex in their composition than the QC samples. The presence of drug-related metabolite(s), co-medication(s), co-medication metabolite(s), dosing vehicle or subject-specific endogenous components might have impact on the trackability of IS for the analyte(s) of interest. In this regard, assay specificity testing should be done as comprehensively as possible during method development. Even with fully validated method, some other factors can introduce variability to IS response in sample analysis, including human error, instrument malfunction or instability. Therefore, it is crucial to routinely monitor IS response in each run to identify abnormality.

A consistent IS response pattern across the knowns (Cs and QCs) and the unknowns for a given study is always a reflection of robustness of the intended method along with solid performance on bench and a well-behaving LC-MS system in the laboratory. In contrast, abnormal IS response variability may be associated with method sub-optimality, study sample specific issue, bench error and/or instrument malfunction. However, depending on how well of the IS tracks the analyte of interest under those 'abnormalities', not all the abnormal IS response variability is associated with inaccuracy of the measured results. The decision tree with a step-by-step procedure introduced in this paper should help identify abnormal IS response variability, guide investigation into the possible root causes and help assessment of the possible impact due to the observed abnormal IS responses.

LC-MS techniques continue evolving. Much has been done in improving the accuracy of autosampler injection volume, robustness of the MS system and others to minimize or eliminate human error in regulated LC-MS bioanalysis. Among the various MS techniques, high-resolution tandem mass spectrometry is a great alternative to complicated and time-consuming method development and optimization in minimizing or eliminating matrix effect or matrix specific interference, the two major contributors to the abnormal IS response variability in LC-MS bioanalysis. While the sensitivity of the current LC-HRMS is less optimal as compared with LC-MRM-MS/MS, with advancement of MS technology, LC-HRMS is expected to deliver the needed sensitivity for the demanding bioanalytical needs.

\section{Financial \& competing interests disclosure}

The authors have no relevant affiliations or financial involvement with any organization or entity with a financial interest in or financial conflict with the subject matter or materials discussed in the manuscript. This includes employment, consultancies, honoraria, stock ownership or options, expert testimony, grants or patents received or pending, or royalties.

No writing assistance was utilized in the production of this manuscript. 


\section{Executive summary}

\section{Background}

- Internal standard (IS) is pivotal to ensure the accuracy and reliability in LC-MS bioanalysis by compensating for the variability of the analyte of interest in bioanalytical workflow. Evaluation, identification and impact assessment of abnormal IS response variability in regulated LC-MS bioanalysis have been emphasized.

IS variability in method development \& pre-study validation

- An IS should have its physicochemical properties very close or identical to the analyte of interest to normalize signal tracking. Thus, a stable-isotope labeled IS (SIL IS) is preferred whenever possible. When SIL IS is not available, a structural analog or other molecule may be used as IS as long as it tracks the analyte of interest.

- An appropriate employment of IS is essential for the robustness of the intended bioanalytical assay. It is important to introduce IS to the workflow as early as possible and thoroughly mix IS with the analyte in biological samples. The selection of IS concentration and/or volume also has impact on IS performance.

- IS performance can be assessed not only from accuracy and precision, but also by selectivity, matrix effect and recovery during method development and pre-study validation.

Common root causes of abnormal IS response variability

- Common root causes of abnormal IS response variability in LC-MS bioanalysis include but are not limited to study subject-specific matrix effect and interferences, instrument/device-related issues, ionization competition between analyte and its SIL IS, spiking errors and instability.

Identification of abnormal IS response variability \& assessment of its impact on LC-MS bioanalysis

- A decision tree was proposed to systematically investigate abnormal IS response variability after each sample analysis run.

- Trend analysis of IS response variability in incurred samples versus known samples is crucial.

- The impact of abnormal IS response could be assessed by simple repeat analysis, analyzing QCs prepared using pre-dose or baseline samples, parallelism and/or standard addition.

Future perspective

- It is important to routinely monitor IS response in each run to identify abnormality. The decision tree with a step-by-step procedure should help identify abnormal IS response variability, guide investigation into the possible root causes and help assessment of the possible impact.

- With advancement of MS technology, LC-HRMS with improved sensitivity is expected to be a great alternative in minimizing or eliminating matrix effect or matrix specific interference, which are the two major contributors to the abnormal IS response variability in LC-MS bioanalysis.

\section{Reference}

Papers of special note have been highlighted as: $\bullet$ of interest; $\bullet \bullet$ of considerable interest

1. Tan A, Boudreau N, Levesque A. Internal standards for quantitative LC-MS bioanalysis. In: LC-MS in Drug Bioanalysis, Xu QA, Madden TL (Eds). 1-32 Springer, Boston, MA, USA (2012).

2. Nilsson LB, Eklund G. Direct quantification in bioanalytical LC-MS/MS using internal calibration via analyte/stable isotope ratio. J. Pharm. Biomed. Anal. 43(3), 1094-1099 (2007).

3. Verhaeghe T. Systematic internal standard variability and issue resolution: two case studies. Bioanalysis 11(18), 1685-1692 (2019).

4. Jian W, Edom RW, Xu Y, Gallagher J, Weng N. Potential bias and mitigations when using stable isotope labeled parent drug as internal standard for LC-MS/MS quantitation of metabolites. J. Chromatogr. B Analyt. Technol. Biomed. Life Sci. 878(31), 3267-3276 (2010).

5. Tan A, Awaiye K. Use of internal standards in LC-MS bioanalysis. In: Handbook of LC-MS Bioanalysis, Li W, Zhang J, Tse FL (Eds). 217-227 John Wiley \& Sons, Inc, NJ, USA (2013).

- A good introduction of internal standards in LC-MS bioanalysis from perspectives of selection, usage, variation and root causes.

6. Stokvis E, Rosing H, Beijnen JH. Stable isotopically labeled internal standards in quantitative bioanalysis using liquid chromatography/mass spectrometry: necessity or not? Rapid Commun. Mass Spectromet. 19(3), 401-407 (2005).

7. Blaye OL. Variations in internal standard response: some thoughts and real-life cases. Bioanalysis 11(18), 1715-1725 (2019).

8. Buonarati MH, Schoener D. Investigations beyond standard operating procedure on internal standard response. Bioanalysis 11(18), 1669-1678 (2019).

9. Fraier D, Ferrari L, Heinig K, Zwanziger E. Inconsistent internal standard response in LC-MS/MS bioanalysis: an evaluation of case studies. Bioanalysis 11(18), 1657-1667 (2019).

10. Tan A, Hussain S, Musuku A, Masse R. Internal standard response variations during incurred sample analysis by LC-MS/MS: case by case trouble-shooting. J. Chromatogr. B Analyt. Technol. Biomed. Life Sci. 877(27), 3201-3209 (2009).

- A good summary on internal standard response variation and associated root causes in LC-MS bioanalysis. 
11. Cho SJ, Vinter S, Garofolo F. Crucial importance of evaluating internal standards (IS) response and troubleshooting in effective LCMS method development, validation and sample analysis. Bioanalysis 11(18), 1645-1647 (2019).

12. Van De Merbel NC, Koster RA, Ohnmacht C. Very complex internal standard response variation in LC-MS/MS bioanalysis: root cause analysis and impact assessment. Bioanalysis 11(18), 1693-1700 (2019).

-• Authors introduced a decisition tree to help identify the root cause for internal standard variability by comparing the responses in study samples versus calibration standards/quality controls.

13. JW E. Learning how to interpret 'dangerous' internal standard behaviors. Bioanalysis 11(18), 1679-1684 (2019).

14. White S, Adcock N, Elbast W et al. European Bioanalysis Forum: recommendation for dealing with internal standard variability. Bioanalysis 6(20), 2767-2774 (2014).

15. US FDA. Evaluation of Internal Standard Responses During Chromatographic Bioanalysis: Questions and Answers (2019). https://www.fda.gov/media/130451/download

16. EMA. Guideline on Bioanalytical Method Validation (2011). https://www.ema.europa.eu/en/documents/scientific-guideline/guideline-bioanalytical-method-validation_en.pdf

17. EMA. ICH Guideline M10 on Bioanalytical Method Validation (2019). https: //www.ema.europa.eu/en/documents/scientific-guideline/draft-ich-guideline-m10-bioanalytical-method-validation-step-2b_en.pdf

18. US FDA. ICH Guideline M10 on Bioanalytical Method Validation (2018). http://academy.gmp-compliance.org/guidemgr/files/UCM368107.PDF

19. Reddy N. Stable labeled isotopes as internal standards: a critical review. Modern Applicat. Pharmacy Pharmacol. 1, (2017).

20. Haubl G, Berthiller F, Krska R, Schuhmacher R. Suitability of a fully ${ }^{13} \mathrm{C}$ isotope labeled internal standard for the determination of the mycotoxin deoxynivalenol by LC-MS/MS without clean up. Anal. Bioanal. Chem. 384(3), 692-696 (2006).

21. Berg T, Strand DH. ${ }^{13} \mathrm{C}$ labelled internal standards-a solution to minimize ion suppression effects in liquid chromatography-tandem mass spectrometry analyses of drugs in biological samples? J. Chromatogr. A 1218(52), 9366-9374 (2011).

22. Fung EN, Aubry A-F, Allentoff A, Ji QC. Utilizing internal standard responses to assess risk on reporting bioanalytical results from hemolyzed samples. AAPS J. 17(5), 1168-1176 (2015).

23. Wu J, Wiegand R, Lorusso P, Li J. A stable isotope-labeled internal standard is essential for correcting for the interindividual variability in the recovery of lapatinib from cancer patient plasma in quantitative LC-MS/MS analysis. J. Chromatogr. B 941, 100-108 (2013).

24. Bakhtiar R, Majumdar TK. Tracking problems and possible solutions in the quantitative determination of small molecule drugs and metabolites in biological fluids using liquid chromatography-mass spectrometry. J. Pharmacol. Toxicol. Methods 55(3), 262-278 (2007).

25. Gu H, Wang J, Aubry AF et al. Calculation and mitigation of isotopic interferences in liquid chromatography-mass spectrometry/mass spectrometry assays and its application in supporting microdose absolute bioavailability studies. Anal. Chem. 84(11), 4844-4850 (2012).

26. Wang S, Cyronak M, Yang E. Does a stable isotopically labeled internal standard always correct analyte response? A matrix effect study on a LC/MS/MS method for the determination of carvedilol enantiomers in human plasma. J. Pharm. Biomed. Anal. 43(2), 701-707 (2007).

27. Lindegardh N, Annerberg A, White NJ, Day NP. Development and validation of a liquid chromatographic-tandem mass spectrometric method for determination of piperaquine in plasma stable isotope labeled internal standard does not always compensate for matrix effects. J. Chromatogr. B Analyt. Technol. Biomed. Life Sci. 862(1-2), 227-236 (2008).

28. Jemal M, Schuster A, Whigan DB. Liquid chromatography/tandem mass spectrometry methods for quantitation of mevalonic acid in human plasma and urine: method validation, demonstration of using a surrogate analyte, and demonstration of unacceptable matrix effect in spite of use of a stable isotope analog internal standard. Rapid Commun. Mass Spectrom. 17(15), 1723-1734 (2003).

29. Liu G, Ji QC, Arnold ME. Identifying, evaluating, and controlling bioanalytical risks resulting from nonuniform matrix ion suppression/enhancement and nonlinear liquid chromatography-mass spectrometry assay response. Anal. Chem. 82(23), 9671-9677 (2010).

30. Wieling J. LC-MS-MS experiences with internal standards. Chromatographia 55(1), S107-S113 (2002).

31. Chavez-Eng CM, Constanzer ML, Matuszewski BK. High-performance liquid chromatographic-tandem mass spectrometric evaluation and determination of stable isotope labeled analogs of rofecoxib in human plasma samples from oral bioavailability studies. $J$. Chromatogr. B Analyt. Technol. Biomed. Life Sci. 767(1), 117-129 (2002).

32. Smith KA, Merrigan SD, Johnson-Davis KL. Selecting a structural analog as an internal standard for the quantification of 6-methylmercaptopurine by LC-MS/MS. J. Applied Lab. Med. 3(3), 384-396 (2018).

33. Wright MJ, Wheller R, Wallace G, Green R. Internal standards in regulated bioanalysis: putting in place a decision-making process during method development. Bioanalysis 11(18), 1701-1713 (2019).

-• Highlights of consideration for internal standard selection and essential experimental assessment for internal standard performance.

34. Takahashi M, Pang H, Kawabata K, Farid NA, Kurihara A. Quantitative determination of clopidogrel active metabolite in human plasma by LC-MS/MS. J. Pharm. Biomed. Anal. 48(4), 1219-1224 (2008). 
35. Bronsema KJ, Bischoff R, Van De Merbel NC. Internal standards in the quantitative determination of protein biopharmaceuticals using liquid chromatography coupled to mass spectrometry. J.Chromatogr. B Analyt. Technol. Biomed. Life Sci. 839-894, 1-14 (2012).

36. Kang L, Weng N, Jian W. LC-MS bioanalysis of intact proteins and peptides. Biomed. Chromatogr. 34(1), e4633 (2020).

37. Bronsema KJ, Bischoff R, Van De Merbel NC. High-sensitivity LC-MS/MS quantification of peptides and proteins in complex biological samples: the impact of enzymatic digestion and internal standard selection on method performance. Anal. Chem. 85(20), 9528-9535 (2013).

38. Van Den Broek I, Niessen WM, Van Dongen WD. Bioanalytical LC-MS/MS of protein-based biopharmaceuticals. J. Chromatogr. B Analyt. Technol. Biomed. Life Sci. 929, 161-179 (2013).

39. Li W, Lin H, Fu Y, Flarakos J. LC-MS/MS determination of a human mAb drug candidate in rat serum using an isotopically labeled universal mAb internal standard. J. Chromatogr. B Analyt. Technol. Biomed. Life Sci. 1044-1045, 166-176 (2017).

40. Fu Y, Li W, Flarakos J. Highly selective and sensitive LC-MS/MS quantification of a therapeutic protein in human serum using immunoaffinity capture enrichment. J.Chromatogr. B Analyt. Technol. Biomed. Life Sci. 1100-1101, 83-90 (2018).

41. Ocana MF, Neubert $H$. An immunoaffinity liquid chromatography-tandem mass spectrometry assay for the quantitation of matrix metalloproteinase 9 in mouse serum. Anal. Biochem. 399(2), 202-210 (2010).

42. Fu Y, Li W, Flarakos J, Tse FLS. Quantitative analysis of pasireotide (SOM230), a cyclic peptide, in monkey plasma using liquid chromatography in combination with tandem mass spectrometry. J. Chromatogr. B Analyt. Technol. Biomed. Life Sci. 1008, 242-249 (2016).

43. Cuadros-Rodriguez L, Bagur-Gonzalez MG, Sanchez-Vinas M, Gonzalez-Casado A, Gomez-Saez AM. Principles of analytical calibration/quantification for the separation sciences. J. Chromatogr. A 1158(1-2), 33-46 (2007).

44. Tan A, Levesque IA, Levesque IM, Viel F, Boudreau N, Levesque A. Analyte and internal standard cross signal contributions and their impact on quantitation in LC-MS based bioanalysis. J. Chromatogr. B Analyt. Technol. Biomed. Life Sci. 879(21), 1954-1960 (2011).

45. Olsson AO, Jelvestam M, Ahnoff M. Variability of the IS revealed ionization enhancement by bile acid in mouse plasma. Bioanalysis 5(19), 2371-2378 (2013).

46. Lindegardh N, Hanpithakpong W, Kamanikom B et al. Major pitfalls in the measurement of artemisinin derivatives in plasma in clinical studies. J. Chromatogr. B 876(1), 54-60 (2008).

47. De Zwart M, Lausecker B, Globig S et al. Co-medication and interference testing in bioanalysis: a European Bioanalysis Forum recommendation. Bioanalysis 8(19), 2065-2070 (2016).

48. Olsson AO, Jelvestam M, Ahnoff M. Variability of the IS revealed ionization enhancement by bile acid in mouse plasma. Bioanalysis 5(19), 2371-2378 (2013).

49. Larger PJ, Breda M, Fraier D, Hughes H, James CA. Ion-suppression effects in liquid chromatography-tandem mass spectrometry due to a formulation agent, a case study in drug discovery bioanalysis. J. Pharm. Biomed. Anal. 39(1-2), 206-216 (2005).

50. Shou WZ, Naidong W. Post-column infusion study of the 'dosing vehicle effect' in the liquid chromatography/tandem mass spectrometric analysis of discovery pharmacokinetic samples. Rapid Commun. Mass Spectrom. 17(6), 589-597 (2003).

51. Liang HR, Foltz RL, Meng M, Bennett P. Ionization enhancement in atmospheric pressure chemical ionization and suppression in electrospray ionization between target drugs and stable-isotope-labeled internal standards in quantitative liquid chromatography/tandem mass spectrometry. Rapid Commun. Mass Spectrom. 17(24), 2815-2821 (2003).

52. Sojo LE, Lum G, Chee P. Internal standard signal suppression by co-eluting analyte in isotope dilution LC-ESI-MS. The Analyst 128(1), 51-54 (2003).

53. Gosetti F, Mazzucco E, Zampieri D, Gennaro MC. Signal suppression/enhancement in high-performance liquid chromatography tandem mass spectrometry. J. Chromatogr. A 1217(25), 3929-3937 (2010). 